\title{
Charge-Remote Fragmentation during FAB-CAD-B/E Linked-Scan Mass Spectrometry of Aminoethyl- Triphenylphosphonium Derivatives of Fatty Acids
}

\author{
Yoon-Seok Chang*.‡ and J. Throck Watson*,† \\ Departments of Biochemistry* and Chemistry,i, Michigan State University, East Lansing, Michigan, USA
}

The carboxyl group of fatty acids is derivatized by aminoethyl triphenylphosphonium (AETPP) bromide. Fast atom bombardment (FAB) collision-activated dissociation (CAD) B/E linked-scan mass spectrometry of these fixed-charge derivatives shows typical charge-remote fragmentation (CRF). Locations of various structural modifications in fatty acids can be recognized easily from CAD spectra of the AETPP derivatives. Because the triphenylphosphonium group localizes positive charge in the molecule, and because a key requirement for CRF is a tightly localized charge site, these preionized molecules fragment under FAB-CAD conditions more effectively than other derivatives that involve ionic bonding with metal cations or protonation of basic sites. Thus, CAD of AETPP derivatives is likely to produce more structurally informative spectra and provide an opportunity to gain additional understanding of the CRF process. The most profound difference between the AETPP derivatives and other cations in positive mode FAB-CAD-B/E-MS is reflected in the substantial improvement of detection limits for the AELPP derivatives over those for the metal cation adducts. For several fatty acids $\left(C_{10}-C_{22}\right)$ tested, the detectability can be enhanced by one to two orders of magnitude when the analysis is performed on the AETPP derivative. In addition, for the analysis of fatty acid mixtures, the FAB mass spectrum of AETPP derivatives produces a relative intensity of the molecular ion peak for each component of the mixture that more closely represents its mole fraction than does that of metal ion adducts. (J Am Soc Mass Spectrom 1992, 3, 769-775)

$\mathrm{C}$ harge-remote fragmentation (CRF) in fast atom bombardment mass spectrometry (FAB-MS) of fatty acids and their metabolities has been studied extensively by Gross and co-workers since 1983 [1] as both a class of gas-phase ion decomposition reactions and a useful method for structure elucidation. FAB collision-activated dissociation tandem mass spectrometry (FAB-CAD-MS/MS) of either carboxylate anions in the negative mode or metal-cationized fatty acids in the positive mode produces diagnostic fragments for recognition of the location of a variety of structural mudifications in these molecules [2]. The fundamental interest in these processes arises from the fact that the fragmentations are initiated neither by a charge nor by a radical site. Labeling studies have shown that these fragmentation reactions produce neu-

\footnotetext{
*New address: Department of Chemistry, 56-028, MIT, Cambridge, MA $02139-4307$

Address reprint request to J. Throck Watson, Department of Biochemistry, Michigan State University, East Lansing, MI 48824-1319.
}

tral alkenes and terminally unsaturated fragment ions as well as hydrogen molecules, presumably through the symmetry-allowed, concerted 1,4-elimination mechanism [3] or, alternatively, through homolytic bond cleavage followed by $\mathrm{H}$ - elimination [4].

Although CRF of fatty acids can be observed when either positive or negative precursor ions are selected, the positive ion mode has a few advantages [5]. The major advantage is the relatively uniform abundance of fragment ions in the FAB-CAD-MS/MS spectra of cationized compounds in comparison to those of carboxylate anions. The cationic fragments also can provide information for determining the position of up to six double bonds [6]. The CAD spectra of [M-H] ions for fatty acids containing more than two conjugated double bonds are difficult to interpret [7]. Alkali or alkaline earth metal ions are most commonly used to localize a positive charge on the molecule. Alternatively, fatty acid derivatives, such as pyrrolidides [8] and picolinyl esters [9], have been prepared to induce $C R F$, in which the derivatized portion of the molecule 
has sufficient proton affinity to localize a charge [10]. The picolinyl ester derivatization, however, has a problem of unwanted chlorination when an excessive amount of thionyl chloride is used with unsaturated fatty acids or some epoxy acids [9].

Even though the FAB-CAD-MS/MS of metal cations or carboxylate anions of fatty acids has been used successfully to elucidate structural features of these molecules, there is a problem that prevents this technique from being successful on a routine basis. Poor ionization efficiency of carboxylate anions and metalcationized species often precludes their use in analyses of small-sized samples by FAB-CAD-MS/MS. The sample size limits for the CAD experiments of the $[\mathrm{M}+2 \mathrm{Li}-\mathrm{H}]^{+}$ions and the $[\mathrm{M}-\mathrm{H}]$ ions were reported to be about $10-50 \mathrm{ng}$ on a triple-sector mass spectrometer [9]. The detection limit was enhanced (about $1 \mathrm{ng}$ ) by using alkaline earth metals for cationization [11]. This enhancement was due primarily to a decrease in matrix ion noise. Our work focuses on the development of an alternative, highly efficient derivatization procedure for positive-charge localization at the carboxylic terminus of fatty acids and on the characterization of the fragmentation behavior of these positive ions during FAB-CAD-MS/MS.

Recently, a simple derivatization method has been used in this laboratory that permits the triphenylphosphonium (TPP) moiety to be selectively attached to the $\mathrm{N}$ - or $\mathrm{C}$-terminus of peptides, thus localizing a positive charge at that site $[12,13]$. The results show that this TPP derivative enhances the detectability of hydrophilic peptides by several orders of magnitude, and generates exclusively and efficiently a complete series of ions from one terminus or the other of the peptide depending on where the TPP function is placed. Because the charge is fixed at either the $\mathrm{N}$ - or C-terminus of peptides, fragment ion formation involves the "charge remote site" process. The C-terminus reaction involves EDC [1-ethyl-3-(3-dimethylaminopropyl)carbodiimide] activation of the terminal carboxylic acid group to permit coupling to the amino group of the aminoethyl-TPP (AETPP) reagent [14]. This method has now been extended to the carboxyl group of fatty acids (Scheme I) [15]. We report here that FAB-CAD$B / E$ linked-scan mass spectra of these fixed-positive
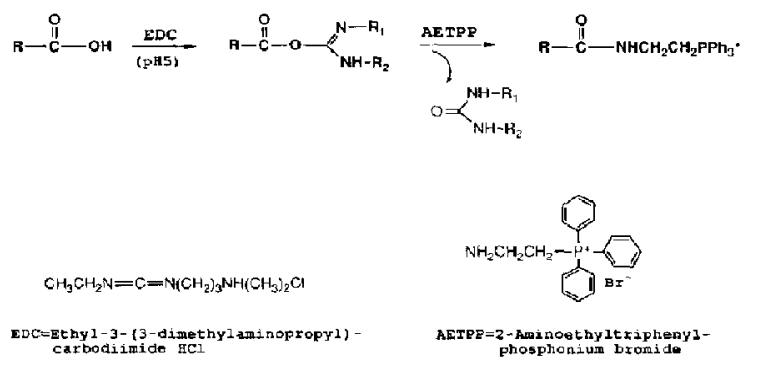

Scheme I charge AETPP-derivatized fatty acids show typical CRF for picogram sample sizes.

\section{Experimental}

Chemicals. Most fatty acids were from Cayman Chemical Co. $(\Lambda \mathrm{nn}$ Arbor, MI) and used without further purification. The fatty acid mixtures were obtained from Supelco (Bellefonte, PA). The aminoethyl-TPP reagent was produced from bromoethyl-TPP in ammonium hydroxide/ethanol solution as described elsewhere [12]. The coupling reagent EDC was obtained from Pierce Chemical Co. (Rockford, IL). The 9-(2'butylcyclopentyl)nonanoic acid was obtained from J. L. Le Quere (Laboratoire de Recherches sur les Aromes, France) as a methyl ester with a purity of about $75 \%$; the methyl ester was converted to a carboxylic acid in alkali solution before the AETP'Pderivatization.

Derizatization reactions. Derivatization of the fatty acid was carried out by dissolving an acid (about $10 \mathrm{ng}$ ) in a micro sample tube containing a dilute trifluoroacetic acid solution (about $0.2 \mathrm{~mL}$ ) adjusting to $\mathrm{pH} 5$. Added to the solution in tenfold excess were the EDC and the AETPP reagents. The reaction mixture was vortexed for 10 to $20 \mathrm{~min}$ and then lyophilized in a vacuum centrifuge tube before analysis by FAB-MS. The reaction efficiency for preparing the AETPP derivatives is estimated to be greater than $95 \%$ based on disappearance of the free acid during the derivatization reaction. The diminution of the free acid was determined by high-performance liquid chromatography and reported as a fraction of the initial concentration of the free acid before the derivatization reaction was initiated.

Fast atom bombardment mass spcctrometry. Solutions of derivatized acids were prepared by dissolving about 10-ng samples in $10 \mu \mathrm{L}$ of acetonitrile solvent. A volume of $1 \mu \mathrm{L}$ of this solution (about $1 \mathrm{ng}$ ) was mixed on the probe tip with about $1 \mu \mathrm{L}$ of matrix, 3-nitrobenzyl alcohol (NBA). Ions were produced with a conventional FAB gun in a JEOL HX-110 double focusing mass spectrometer operated in the positive ion mode. The accelerating voltage was $10 \mathrm{kV}$ and the resolution was set at 3000 (50\% valley). For FAB-CAD$B / E$ spectra, helium was used as the collision gas in a cell located in the first field-free region. The helium pressure was adjusted to reduce the abundance of the parent ions by $50 \%$. The JEOL DA-5000 data system generated linked scans at constant $B / E$ ratio. Typically, data were collected from $m / z 300$ to avoid peaks representing the 'I'P'P moiety and to emphasize those representing structural features of the parent compound. Three scans were averaged for the CAD spectra. Metal cationization was accomplished by mixing on a probe tip the fatty acids with NBA saturated with barium hydroxide. The FAB-CAD-MS/MS of a 


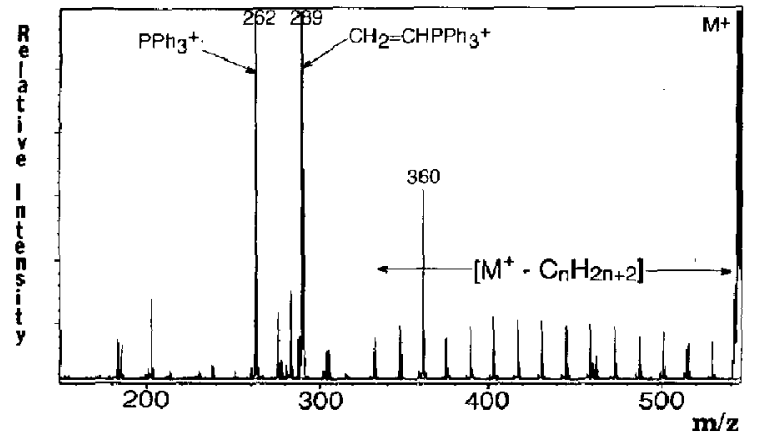

Figure 1. FAB-CAD-B/E spectrum of the AETPP derivative of palmitic acid, $m / z\left[\mathbf{M}^{+} \mid 544\right.$.

fatty acid mixture was carried out with a tandem highresolution mass spectrometer (JEOL, HX110/HX110) at the Massachusetts Institute of Technology.

\section{Results and Discussion}

Conventional FAB-MS of the positive ions of AETPPderivatized fatty acids provides little or no information regarding structure because desorption is accompanied by little fragmentation except for loss of alkyl-TPP moieties. However, the CAD spectrum of the $\mathrm{M}^{+}$ion of AETPP derivatives shows remote charge site fragmentation, as well as fragmentation proximate to the charge site (Figure 1). The characteristic peaks at $\mathrm{m} / \mathrm{z}$ $262\left(\mathrm{PPh}_{3}^{+}\right)$and $289\left(\mathrm{CH}_{2}=\mathrm{CHPPh}_{3}^{+}\right)$represent the latter process. These abundant cations are characteristic of decompositions of most AETPP-derivatized carboxylic acids. The CRFs result in parallel losses of $\mathrm{C}_{n} \mathrm{H}_{2 n \mid z}$ units beginning at the alkyl terminus.* This characteristic fragmentation pattern resembles those found for carboxylate anions and metal adduct cations of saturated fatty acids [16] and picolinyl ester derivatives [9]. The peak at $m / z 360$ in the CAD spectrum of AETPP derivatives is noticeably large because the formation of the $\alpha, \beta$-unsaturated kefone is thermolytically favored (Scheme II). Some examples of the utility of the FAB-CAD-B / E linked-scan spectra for structural characterization of AETPP-derivatized fatty acids are presented below.

The methyl branch positions in 4,8,12-trimethyloctadecanoic acid are easily recognized from the C.AD<smiles>CCc1ccc(C(O)CC)cn1</smiles><smiles>CC(C)C</smiles><smiles>C=CCC</smiles><smiles>C=CC(=O)CC</smiles>

Scheme II

\footnotetext{
- The origin for the ion of mass 462 is not clear at this time. We assume this to be an impurity (possibility from the matrix) due to the B/E's low-resolution patent ion selection. This peak diminished as a larger amount of sample was used to generate the parent ion for CAD.
}

spectrum of the $\mathrm{M}^{+}$ion of the AETPP derivative (Figure 2a). The charge remote decompositions involve parallel pathways of losses of $\mathrm{C}_{n} \mathrm{H}_{2 n+2}$ groups differing by one carbon except for fragmentations occurring at the branch points, where the significant losses of $\mathrm{C}_{n} \mathrm{H}_{2 n+2}$ groups differ by two carbons, causing an obvious interruption in the main series of peaks. Thus, the presence of three branch points is clear from the 28-u gaps in the pairs of relatively large peaks $a$ and $b$, $c$ and $\mathbf{d}$, and $\mathbf{e}$ and $\mathbf{f}$. Iso- and anteiso-branched fatty acids are also easily distinguished by this characteristic fragmentation pattern. The spectrum of the isobranched acid (Figure 2b) shows the absence of a peak at $m / z 514$ that would otherwise correspond to loss of $\mathrm{C}_{2} \mathrm{H}_{6}$. The anteiso-branched acid (Figure 2c), on the other hand, fragments to undergo losses of $\mathrm{CH}_{4}, \mathrm{C}_{2} \mathrm{H}_{6}$, and $\mathrm{C}_{4} \mathrm{H}_{10}$, but virtually no loss of $\mathrm{C}_{3} \mathrm{H}_{8}$ occurs. Thus,
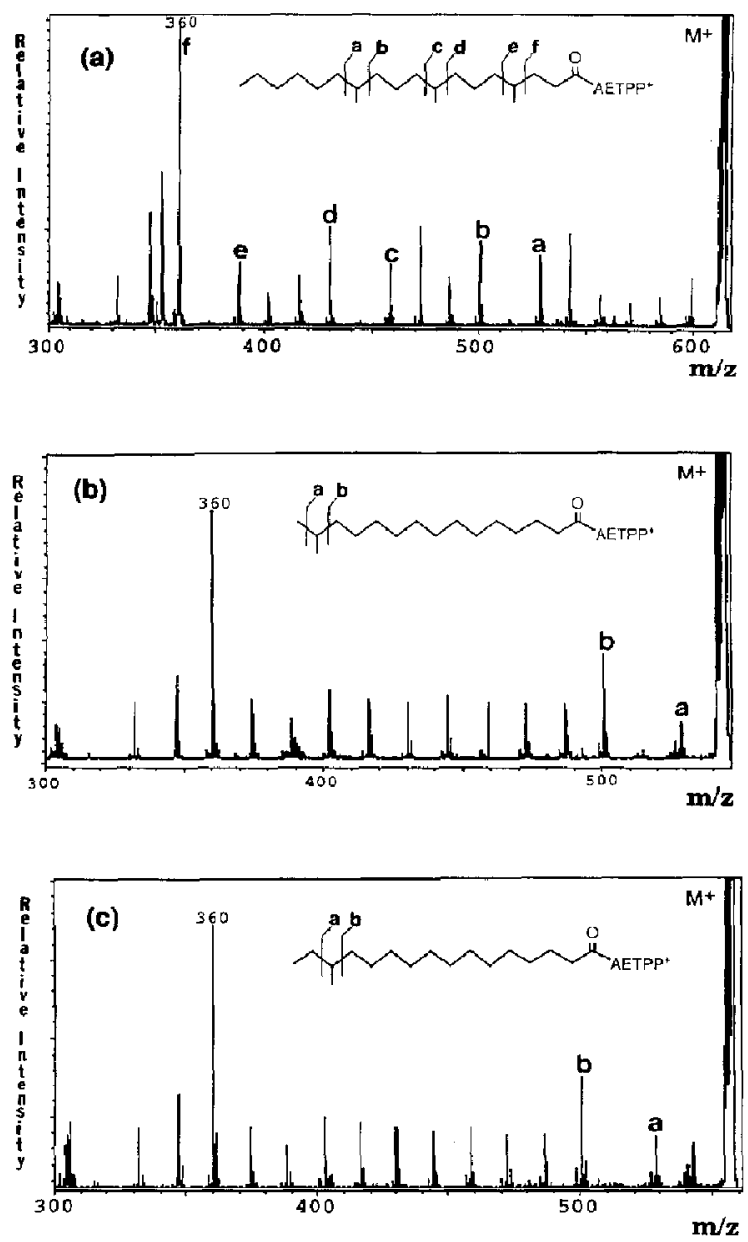

Figure 2. FAB-CAD-B/E spectra of the AETPP derivatives of (a) 4,8,12-trimethyloctadecanoic acid $\left(m / z: M^{+}\right.$614, a 528, b 500 , c 458, d 430, e 388, f 360), (b) isopalmitic acid ( $\mathrm{m} / \mathrm{z}: \mathrm{M}^{+}$ 544 , a 528 , b 500$)$, and (c) anteisomargaric acid $\left(m / z: M^{+} 558\right.$, a $528, b$ 500). 
the location of methyl branches in iso- and anteiso-fatty acids can be recognized easily from FAB-CAD-B/E spectra of AETPP derivatives, in a manner analogous to that based on CRF of the free acid [9].

Collisional activation of the AETPP derivatives of polyunsaturated fatty acids induces the expected CRF as interrupted by the double bonds. The CAD spectrum of AETPP-derivatized arachidonic acid (Figure 3a), for example, shows simple allylic cleavages on the alkyl-terminal side of the double bond. Establishing the location of double bonds, therefore, is straightforward. The AETPP spectrum also shows that vinyl bond cleavage appears to be more facile than double bond cleavage; this is hardly seen in the spectrum of the barium adduct [11]. In general, these covalently bonded AETPP cations have an advantage over the metal-adduct cations, for which metal ion attachment to the double bond induces abundant loss of water during analysis. Preparation of the AETPP derivative of docosahexaenoic acid containing six double bonds has been achieved without isomerization of the double bonds; analysis of this derivative by FAB-CAD-B/E shows fragmentations (Figure $3 b$ ) that are distinctly indicative of double bond positions and are comparable to those of the lithium adduct [6]. The AETPP derivatization procedure also is applicable to epoxysubstituted eicosatrienoic acid without disturbing the epoxy group during the derivatization reaction. The presence of an epoxy substituent in the polyunsaturated acid can be recognized from the spectral region
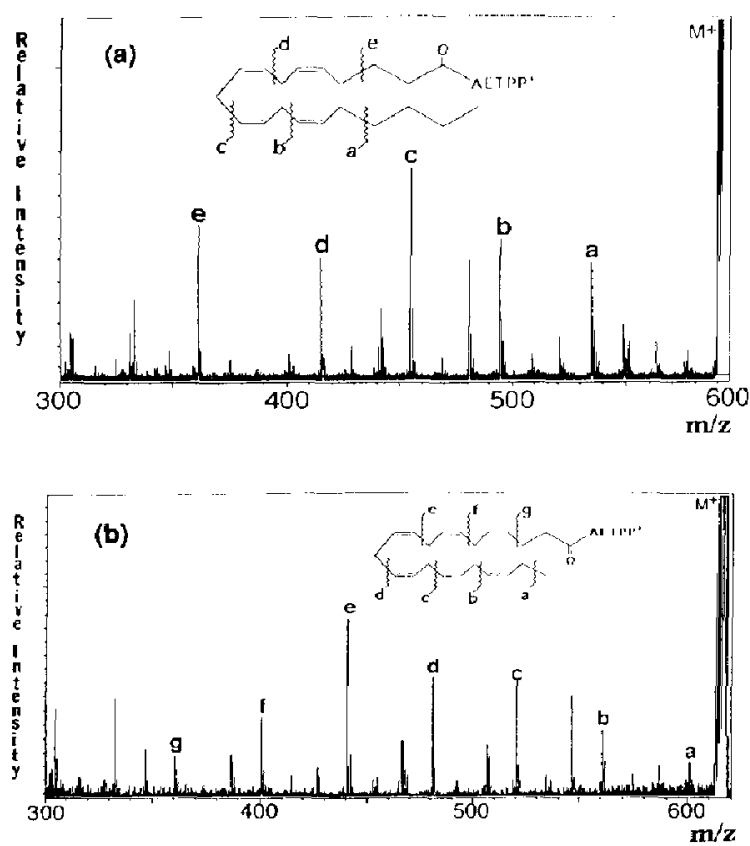

Figure 3. FAB-CAD-B/E spectra of the AETPP derivatives of (a) arachidonic acid $\left(m / z: \mathrm{M}^{+} 592\right.$, a 534, b 494, c 454 , d 414 , e 360) and (b) docosahexaenoic acid ( $m / z \mathrm{M}^{+} 617$, a $600, \mathbf{b} 560$, c 520 , d 480 , e 440 , f 400 , g 360 ). having two intense peaks with a gap of $41 \mathrm{u}$ (Figure 4).

The AETPP methodology was applied to the structural characterization of cyclic fatty acids that are formed in fats and oils during deep fat frying [17]. These cyclic fatty acids, which may be toxic, usually exist as complex mixtures of various isomers; characterization of specific compounds has been achieved partly by gas chromatography mass spectrometry (GCMS) or GC-MS/MS of pentafluorobenzyl ester derivatives [18]. The 1,2-disubstituted cyclopentyl acid isolated from heated fats was derivatized and the FAB-CAD-B/E spectrum of the AETPP derivative (Figure 5) shows CRF of the alkyl chain with more facile cleavages at points a and $e$, which correspond to fragmentation adjacent to the cyclopentane ring. The number of clusters of regularly spaced peaks between peak $e$ and the characteristic peak at $\mathrm{m} / \mathrm{z} 360$ (as shown in Scheme II, this fragment includes the first two carbons of the internal $n$-alkyl chain) can be related to the number of carbon atoms in the internal n-alkyl substituent. As was observed for the other fatty acids, the major advantage of the CAD spectrum of the AETPP derivative is the relatively uniform abundance of fragmentation between the internal and terminal alkyl chains. Furthermore, fragmentation leading to the unexplained ion described by Le Quere et al. (peak c between peaks $a$ and $b$ in ref 18) in the CAD spectrum of the carboxylated anion is substantially suppressed in the fragmentation of the AETPP derivative, so that the presence of the cyclopentane ring can be more easily recognized. The two small peaks $c$ and $\mathrm{d}$ in Figure 5 correspond to the $\mathrm{C}-\mathrm{C}$ bond cleavages of the cyclopentane ring. 'The origin of peak $\mathbf{b}$ at $\mathrm{m} / \mathrm{z}$ 494, however, is not yet clear.

Efficiency of AETPP derivatization is a principal objective in our ongoing study to develop a methodology that can be utilized readily in the analysis of picogram quantities of fatty acids isolated from biological sources. Because we could not reproduce the detection limit of $1 \mathrm{ng}$ for barium-cationized acid with our instrument as was reported by Davoli and Gross.

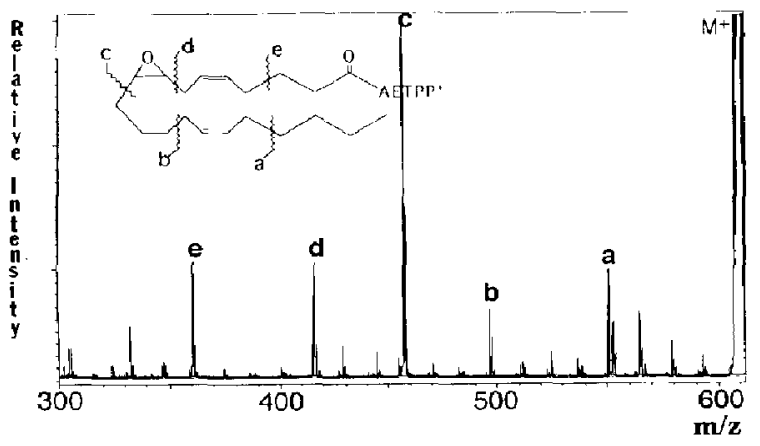

Figure 4. FAB-CAD-B / E spectrum of the AETPP derivative of $8(9)$ :-epoxyeicosatrienoic acid $\left(m / z: \mathrm{M}^{+}\right.$609, a 550 , b $496, \mathrm{c} 456$, d 415, e 360). 


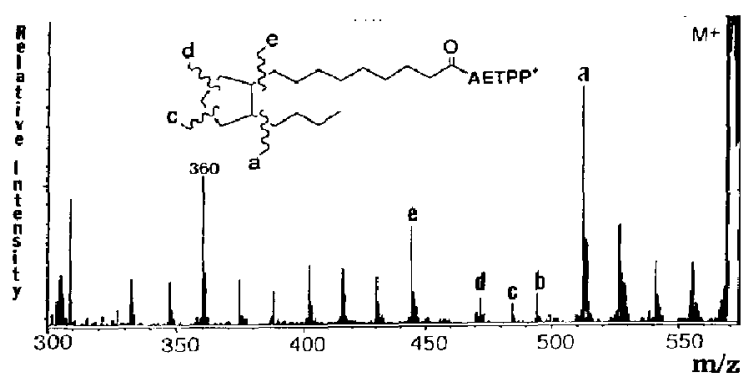

Figure 5. FAB-CAD-B/E spectrum of the AETPP derivative of 9-(2'-butylcyclopentyl)nonanoic acid $\left(m / z ; \mathbf{M}^{+} 570\right.$, a 512, b 494, c 485, d 472, e 444). The number of peak clusters between peak $e$ and that at $m / z 360$ can be related to the number of carbons in the internal n-alkyl chain; peak e represents an ion containing all eight carbons of the internal n-alkyl chain.

[11], we compared the relative sensitivity of the two methods. The barium adducts afford cationization of the analytes with formation of $[\mathrm{M}-\mathrm{H}+\mathrm{Ba}]^{+}$and enhance detection relative to that of lithium adducts. However, the AETPP derivatives improve the analysis of fatty acids to an even greater extent. Figure 6, for example, represents a greater than fiftyfold increase in detectability of the AETPP derivative of pentadecanoic acid compared to that of the barium adduct. This enhancement factor was assessed by comparing the intensities of peaks corresponding to the barium adduct and the molecular ion of the AETPP derivative under
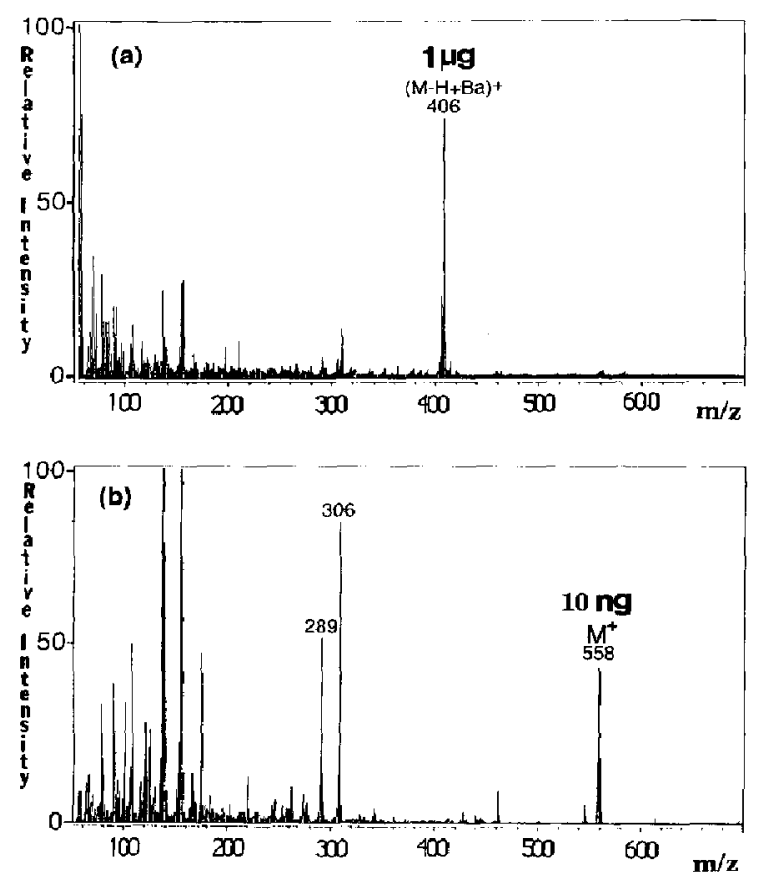

Figure 6. FAB mass spectra of (a) the barium adduct $(\mathrm{m} / \mathrm{z}$ $\left.[\mathrm{M}-\mathrm{H}+\mathrm{Ba}]^{+} 406\right), 1 \mu \mathrm{g}$ and (b) the AETPP derivative $(m / z$ $\left.\left[\mathrm{M}^{+}\right] 558\right), 10 \mathrm{ng}$ of pentadecanoic acid. the same FAB conditions and without subtracting the background. The observed peak intensity (profile data) of the AETPP derivative is approximately 50 times larger than that of the barium adduct with compensation of sample quantity. The detection limit for the $\mathrm{M}^{+}$ ion of AEIPP-derivatized oleic acid was as low as 0.1 $\mathrm{ng}$ at a signal-to-noise ratio of about 5 to produce an interpretable CAD spectrum. The result was obtained in spite of matrix interference encountered when analyzing small amounts of the AETPP derivative in the presence of excess AETPP-derivatizing reagents. Much higher sensitivity was accomplished for the CAD spectrum of the AETPP derivative of oleic acid than for the barium adduct; in Figure 7, the B/E linked-scan spectrum of the AETPP derivative from $0.1 \mathrm{ng}$ of oleic acid $(\mathrm{m} / z 570)$ is compared with that of the barium adduct ( $m / z$ 419) from a 10-ng sample. Future experiments should produce more favorable results after separating excess derivatizing reagents from derivatized analytes with a chromatographic procedure before or during the FAB-CAD experiments.

Another goal of our developing methodology is to apply the AETPP derivatizing reaction to mixtures of fatty acids. One of the first questions to address is the possible difference in reaction rates between individual acids in the mixture. Another important factor, particularly for the unstable acids involving one or more functional groups, is the possiblity for side reactions, which may not be significant in the derivatization of simple, saturated fatty acids. Figure 8 illustrates preliminary results obtained with a commercially
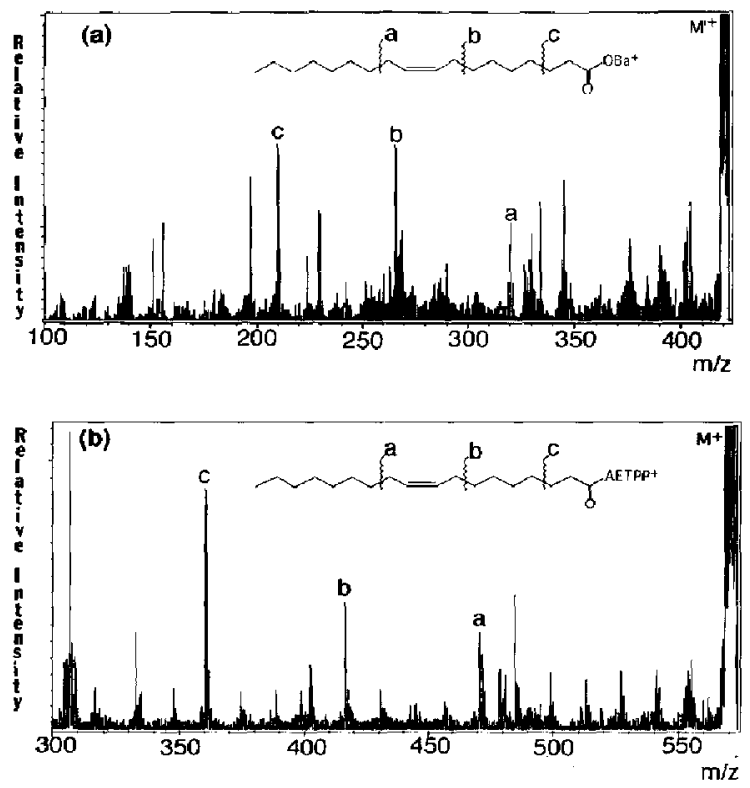

Figure 7. FAB-CAD-B $/ E$ spectra of (a) the barium adduct $(m / z$ $\left.[\mathrm{M}-\mathrm{H}+\mathrm{Ba}]^{+} 419\right), 10 \mathrm{ng}$ and $(\mathrm{b})$ the AETPP derivative $(\mathrm{m} / \mathrm{z}$ $\left.\left(\mathrm{M}^{+}\right) 570\right), 0.1 \mathrm{ng}$ of oleic acid. The spectra were obtained with a resolution of $\mathbf{1 0 0 0}$. 

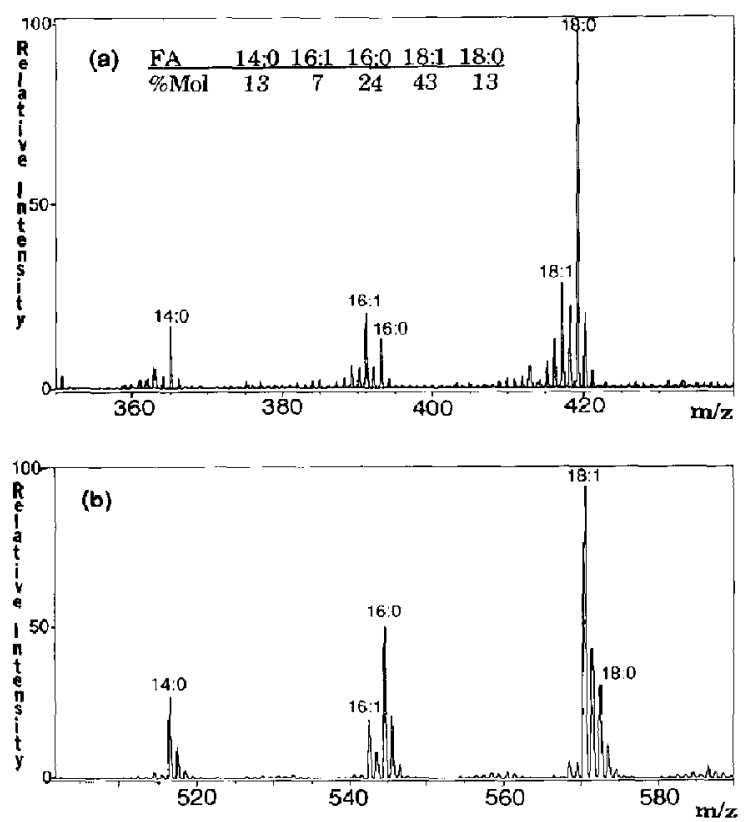

Figure 8. FAB mass spectra of (a) the barium adducts and (b) the AETPP derivatives of fatty acid mixture (A-NHI-D).

available fatty acid mixture (A-NHI-D, Supelco, Bellefonte, PA). The barium adduct $[\mathrm{M}-\mathrm{H}+\mathrm{Ba}]^{+}$of each of the fatty acid components is observed in the FAB mass spectrum. However, the relative intensity of the molecular adducts $[\mathrm{M}-\mathrm{H}+\mathrm{Ba}]^{+}$does not reflect the molar ratio of the fatty acids in the mixture. For example, the relative intensity of $[\mathrm{M}-\mathrm{H}+\mathrm{Ba}]^{+}$for the fatty acids 18:1 and 18:0 is approximately 1:3.6 (Figure 8a); the actual molar percent composition of the mixture is shown on the upper panel of figure, indicating that the molar ratio of 18:0 to 18:1 should be 3.3:1 (this was confirmed by treating an aliquot of the mixture with $\mathrm{CH}_{2} \mathrm{~N}_{2}$ and analyzing it by gas-liquid chromatography with flame ionization detector). Following AETPP derivatization, on the other hand, analysis by $F A B$ produces a mass spectrum in which the relative intensity of the molecular ion peak for each component of the mixture more closely represents its mole fraction in the fatty acid mixture; the peak intensity of $18: 1$ to the peak intensity of 18:0 is ca. 3.2:1 (Figure 8b). Similar results also were obtained with the other fatty acid mixture standards (A-NHI-A, B, C, E, F).

Because this sample mixture contains fatty acids differing by only two mass units (e.g., 16:1 and 16:0, $18: 1$ and 18:0), correct interpretation of the fragmentation patterns requires that the individual analytes should be separated by some procedure. One approach would be to employ a chromotographic separation step prior to mass spectral analysis. This could be accomplished either with off-line or on-line liquid chromatography mass spectrometry (LC-MS). We ex- pect that the triphenylphosphonium group in the AETPP moiety of the molecule will increase the molar absorptivity of the derivatized fatty acids at a wavelength different from that of the background, allowing detection of microquantities. A second stage of this work will be directed toward the on-line separation and analysis of the derivatized fatty acid mixtures by LC-MS. An alternative technique is the analysis of individual analytes in the mixture by tandem mass spectrometry, such as the use of a four-sector mass spectrometer to select individual $\mathrm{M}^{+}$ions of the different fatty acid derivatives for CAD analysis. The parent ion resolution of the four-sector instrument permits diagnostic daughter ion spectra to be required for components differing by 2 u (Higure 9); this is not possible using linked scanning at constant $B / E$ in a two-sector instrument.

\section{Conclusion}

In conclusion, we have developed a new method to localize a charge on fatty acids before analysis by FAB-CAD by introducing the cationic triphenylphosphonium group at the carboxylic end of molecule. This method allows the charge site to be localized in the molecular ion, yielding CRF without scrambling of the charge location. For example, the FAB-CAD-B/E spectrum (Figure 10) of the AETPP derivative of cholesteryl hemisuccinate shows that the alkyl-terminal decomposition sites are completely isolated from the charge site in the hemisuccinate moiety by a rigid group of inter-
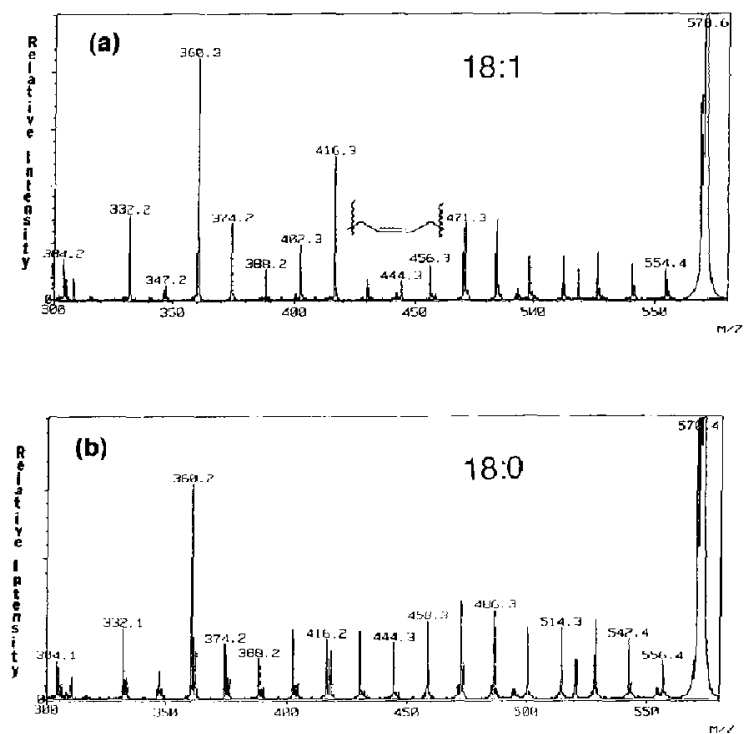

Figure 9. FAB-CAD-MS/MS spectra of the AETPP derivatives of (a) fatty acid 18:1 $\left(\mathrm{m} / \mathrm{z}\left[\mathrm{M}^{+}\right] 570\right)$ and (b) fatty acid 18:0 $(\mathrm{m} / \mathrm{z}$ $\left.\left[\mathrm{M}^{+}\right] 572\right)$. The individual $\mathrm{M}^{+}$ions were selected during analysis of the fatty acid mixture (A-NHI-D) by MS- $[$ of a tandem mass spectrometer (JEOL, HX110/HX110, MIT). 


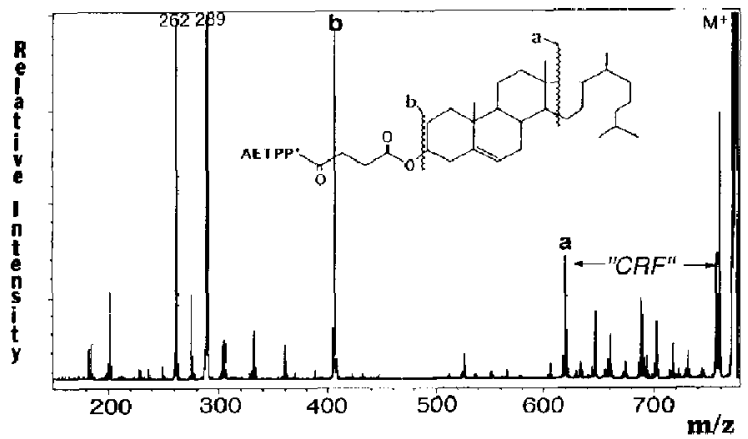

Figure 10. FAB-CAD-B/E spectrum of the AETPP derivative of cholesteryl hemisuccinate $\left(m / z: \mathrm{M}^{+} 775\right.$, a 619, b 405).

nal cyclohexane rings, which is represented by the region between $\mathbf{a}$ and $\mathbf{b}$ in the spectrum. This method should be useful for differentiation of positional or stereochemical isomers [19]. Because the triphenylphosphonium group localizes the positive charge, and because a key requirement for CRF is a tightly localized charge site, these preionized molecules may fragment under FAB-CAD conditions more effectively than other derivatives that involve ionic bonding with metal cations or protonation of basic sites. Thus, CAD of AETPP derivatives is likely to produce additional structurally informative spectra and provide an opportunity for greater understanding of the CRF process.

The most profound difference between the spectra of the AETPP derivatives and those of cation adducts in positive mode FAB-CAD-B/E-MS involves the substantial improvement in detection limits for the AETPP derivatives. For several fatty acids $\left(\mathrm{C}_{10}-\mathrm{C}_{22}\right)$ tested, the detectability can be enhanced by one to two orders of magnitude. This enhancement is mainly due to the high efficiencies in both derivatization reactions and FAB-desorption processes for AETPP derivatives. As discussed for alkaline earth metal ion adducts earlier [11], this may also be due, in part, to the fact that the preionized AETPP derivatives are shifted up in mass by $288 \mathrm{u}$ into a region where the chemical noise is lower. On the basis of these preliminary results, AETPP derivatization of arachidonic acid metabolites is worthy of in-depth investigation for advantages in identifying and characterizing structural features of this im- portant class of compounds. The application of this procedure to arachidonic acid metabolites is currently under investigation.

\section{Acknowledgments}

This work was supported in part by the Michigan State University Research Excellence Fund with equipment made available in the MSU Mass Spectrometry Facility as supported by a grant (RR-0480-24) from the Biomedical Resource Technology Program of the National Center for Research Resources of the National Institutes of Health. We thank D. A. Gage and Z. H. Huang for helpful discussions. Thanks also go to C. E. Costello at M.I.T. for analysis of fatty acid mixtures by a tandem mass spectrometer and to J. L. Le Quere for providing cyclic tatty acids.

\section{References}

1. Tomer, K. B.; Crow, F. W.; Gross, M. L. J. Am Chem. Soc. 1983, 105, 5487.

2. Jensen, N. J.; Gross, M. L. Mass Spectrom. Rev. 1987, 6, 497.

3. Contado, M. J.; Adams, J.: Jensen, N. J.; Gross, M. L. J. Am. Soc. Mass Spectrom. 1991, 2, 180.

4. Wysocki, V. H.; Ross, M. M. Int. J. Mass Spectrom. Ion Proc. 1991, 104, 179.

5. Crockett, J. S.; Gross, M. L.; Christie, W. W.; Holman, R. T. J. Am. Soc. Mass Spectrom. 1990, 1, 1983.

6. Adams, J-; Gross, M. L. Attal. Chett. 1987, 59, 1576.

7. Jensen, N. J.; Tomer, B.; Gross, M. L. Anal. Chem. 1985, 57, 2018.

8. Vetler, W.; Meister, W. Org. Mass Spectrom 1986, $21,85$.

9. Deterding, L. J.; Gross, M. L. Anal. Chim. Acta 1987, $200,431$.

10. Christie, W. W.; Brechany, E. Y.; Johnson, S. B.; Holman, R. T. Lipids 1986, 21,657.

11. Lavoli, E.; Gross, M. L. I. Am Soc. Mass Spectrom. 1990, 1, 320.

12. Wagner, D. S.; Salari, A.; Fetter, J.; Gage, D. A.; Leykam, J.; Hollingsworth, R.; Watson, J. T. Biol. Mass Spectrom. 1991, 20, 419.

13. Watson, J. T.; Wagner, D. S.; Chang, Y. S.; Strahler, J.; Hanash, S.; Gage, D. A. Int. J. Mass Spectrom. Ion Proc. 1991, 111, 191.

14. Swan, J. M.; Wright, S. H. B. Aust. J. Chem. 1971, 24, 777.

15. Chang. Y. S.; Watson, J. T. Presented at The 39th ASMS Conference on Mass Spectrometry and Allied Topics, Nashville, TN, 1991.

16. Adams, 1.; Gross, M. L. Org. Mass Spectrom. 1988, 23, 307.

17. Potteau, B. Ann. Nutr. Aliment. 1976, 30, 67.

18. Le Quere, J. L.; Sebedio, J. L.; Henry, R.; Couderc, F.; Demont, N.; Prome, J. C. I. Chromatogr. 1991, 562, 659.

19. Tomer $r$ K. B.; Gross, M. L. Biomed. Environ. Mass Spectrom. $1988,15,89$. 